\title{
Influenza Vaccination and Healthcare Personnel Compliance
}

\author{
Megan C. Gallagher, $M D, M P H^{1, *}$ \\ Sarah Haessler, $M D^{1}$ \\ Hilary M. Babcock, MD, $\mathrm{MPH}^{2}$
}

\author{
Address \\ ",1Division of Infectious Disease, University of Massachusetts Medical \\ School-Baystate, Springfield, MA, USA \\ Email: megan.gallagher@baystatehealth.org \\ ${ }^{2}$ Division of Infectious Diseases, Washington University School of Medicine, St \\ Louis, MO, USA
}

Published online: 24 February 2020

(C) Springer Science+Business Media, LLC, part of Springer Nature 2020

This article is part of the Topical Collection on New Technologies and Advances in Infections Prevention

Keywords Influenza vaccination - Mandatory vaccine policies $\cdot$ Healthcare personnel vaccination - Presenteeism . Masking requirements

\begin{abstract}
Purpose of Review We reviewed the benefit of influenza vaccination in healthcare personnel (HCP), rates of vaccine coverage, and practices used to try to boost vaccine coverage among HCP.

Recent Findings Influenza vaccination in HCP provides benefits to both HCP and patients, including reductions in patient morbidity and mortality and decreases in HCP absenteeism. Despite these benefits, influenza vaccine coverage among HCP still falls short of the Healthy People 2020 goal of $90 \%$. As a result, healthcare institutions have used various practices to boost vaccination, including mandatory vaccine policies and requiring nonimmunized HCP to wear masks during the influenza season. All of these efforts have been successful at increasing vaccination rates, and employer vaccination requirements have led to rates that meet the Healthy People 2020 goal. Rates of mandatory vaccine policies have increased over time, and several states now have influenza vaccine requirements. However, additional study into how these policies improve patient outcomes is needed. Summary Continued effort is needed to boost influenza vaccination rates among HCP, and mandatory vaccine policies may be used if other methods have not been effective in adequately raising vaccination rates. Future research should focus on how mandatory vaccine policies can improve patient outcomes.
\end{abstract}




\section{Introduction}

Annual vaccination is one of the mainstays of influenza prevention and decreases morbidity and mortality from influenza. Currently, the Advisory Committee on Immunization Practices (ACIP) recommends that all persons over the age of 6 months without contraindications receive an annual influenza vaccine [1•]. Given the beneficial impact on the general population, annual influenza vaccination of healthcare personnel (HCP) has been recommended by the ACIP since 1984 [2], and the Healthy People 2020 campaign set a target of $90 \%[3]$.

There are no recommendations around which specific vaccine should be used, although inactivated vaccine is recommended over live attenuated vaccine for immunocompromised patients $[1 \bullet]$. A history of GuillainBarré syndrome within 6 weeks of a previous dose of influenza vaccine is a precaution, and generally, persons with this history should not be vaccinated. Patients with a history of egg allergy can generally receive any licensed, recommended vaccine, as the amount of egg protein is very small, but persons with a history of severe reactions to egg should receive vaccination under the supervision of a healthcare provider who can manage allergic reactions or receive a vaccine whose formulation is egg free, such as the recombinant vaccine. A previous severe allergic reaction to influenza vaccine, regardless of the type of vaccine, is a contraindication [1•].

Vaccine effectiveness varies from year to year due to antigenic drift by circulating influenza viruses. However, even in years in which the vaccine effectiveness is lower than expected, vaccination has been shown to prevent influenza-related illnesses. In the 2017-2018 influenza season, the vaccine effectiveness was calculated to be $38 \%$ [4]. Despite this poor match, influenza vaccination was still estimated to prevent 7.1 million illnesses, 3.7 million medical visits, 109,000 hospitalizations, and 8000 deaths [4].

Currently, the ACIP recommends offering vaccination by the end of October [1•]. The optimal timing of influenza vaccination is challenging-while vaccination should occur prior to the onset of influenza season, the timing of each individual season varies year to year. There is some evidence to suggest that the effectiveness of influenza vaccine wanes during the course of the influenza season, and that the more time that elapses since vaccination, the risk of influenza infection increases [5]. However, there is also concern that delaying vaccination campaigns may lead to missed opportunities to vaccinate. Regardless of when vaccination starts, it should continue to be offered through the influenza season as it may still be beneficial $[1 \bullet]$.

A randomized controlled trial performed in the 1990s found that influenza vaccination of HCP was effective in preventing serologically defined infection with an efficacy of $88-89 \%$ [6]. The primary benefit of HCP vaccination is personal protection against influen$\mathrm{za}$, but this can lead to the secondary benefit of protecting patients from influenza infections transmitted by HCP. An observational study among elderly patients in long-term care found that HCP vaccination decreased patient mortality, but vaccination of patients was not associated with the same decrease in mortality, likely due to inadequate immune response after vaccination by the patients [7]. Cluster randomized trials in long-term care facilities found that increases in HCP vaccination led to decreases in mortality $[8,9]$, influenza-like illness [9], and hospitalizations for influenza-like illness [9]. While no randomized trials have been performed in the acute care setting, similar findings have been noted in observational studies. In two observational studies, increasing the HCP influenza vaccination rate was associated with a significant decrease in the rate of nosocomial influenza infections at two separate acute care hospitals $[10,11]$. The converse phenomenon has also been observed, in which a decrease in HCP influenza vaccination from 13.2 to $3.2 \%$ over a 7 -year period was associated with a significant increase in the rate of nosocomial influenza infections in an acute care hospital [12]. A case-control study of nosocomial influenza found a median HCP vaccination rate of $11.5 \%$ on the units with the nosocomial influenza cases and a median rate of $36.1 \%$ on the units with the controls [13].

One explanation for the effectiveness of HCP vaccination in preventing cases of nosocomial influenza is likely tied into the concept of presenteeism, in which ill employees come to work while sick. Multiple surveys of HCP have revealed that the majority of respondents come to work when sick with a respiratory infection [14-17]. Commonly reported reasons for working while 
sick included obligations to colleagues, obligations to patient care, and sick leave structures that can end up penalizing HCPs for taking time off $[14,17,18]$. Most of these surveys have focused on physicians, but a study in a long-term care facility also found $89 \%$ of the HCPs with acute respiratory infections worked while ill [19]. Depending on workplace policies, HCPs may have limited allocated sick days and may feel a financial incentive to work while sick. A survey regarding pandemic influenza mitigation recommendations found that $28 \%$ of respondents felt that they would lose their job if they had to stay home for 7 to 10 days, and those who earned less than $\$ 75,000$ per year were more likely to report some degree of financial hardship from staying home [20].

Even if presenteeism is decreased, asymptomatic viral shedding can still lead to influenza transmission. Depending on how asymptomatic infections are defined, they range from 4 to $28 \%$ in outbreak settings [21]. Asymptomatic viral shedding has been reported [22], and the full extent to which it leads to transmission is still not well understood [23]. However, people with only mild symptoms could easily come to work and be infectious.

Another potential secondary benefit of HCP vaccination is reducing the rate of absenteeism secondary to influenza illness. Once British Columbia moved to a province-wide mandatory HCP vaccination or masking policy, they found that HCP who were vaccinated early took less sick time than those who were vaccinated late or not at all [24]. In an observational study of multiple health care systems, systems with mandatory vaccination policies had lower rates of symptomatic absenteeism [25]. A randomized controlled trial in pediatric hospitals showed that influenza vaccination did not decrease the number of episodes or days of respiratory infections, but it did decrease the number of days of work lost due to respiratory infection [26]. Reducing the rate of absenteeism can have a significant impact on patient care. A nosocomial influenza outbreak in one unit of one acute care hospital in France led to 14 person-days of sick leave, postponing 8 scheduled admissions, suspending emergency admissions for 11 days, and an increased average charge per infected patient of $\$ 3798$ [27].

Despite the ACIP recommendations and the evidence of benefit in reducing nosocomial influenza, national influenza vaccination rates among HCP still fall short of the 90\% goal [5]. In the 2017-2018 influenza season, $78.4 \%$ of HCP reported having received a vaccine [28•]. The rate has increased from the 20102011 season but has been flat over the past 4 influenza seasons. Vaccination rates are highest among hospitals, followed by ambulatory/office settings, and lowest in the long-term care setting [28•]. Physicians have the highest vaccination rates, followed by pharmacists, nurses, and nurse practitioners/physician assistants, while non-clinical personnel and aides have the lowest rates [28 $\bullet$. When HCP are surveyed about their reasons for declining vaccination, common responses include a perception that the vaccine is unsafe, a perception that the vaccine is unreliable, fear of adverse effects, lack of time to get a vaccine, and forgetting to get a vaccine [29]. A variety of methods have been used to improve vaccine uptake among HCP, specifically targeting these issues, including educational campaigns, tracking rates of vaccine uptake, mobile vaccine carts, mandatory declination, peer-to-peer vaccination efforts, and vaccination stations at healthcare facility entrances [10, 11, 30, 31].

Given this, many health care organizations and some states are moving towards mandatory vaccination policies. The types of mandatory vaccine policies vary around the consequences for not getting vaccinated and the types of exemptions that are allowed. In a 2017 survey, among 368 hospitals with a vaccine mandate, $82.9 \%$ required non-vaccinated employees to wear a mask when providing patient care during influenza season and $74.3 \%$ had penalties for non-compliance with influenza vaccination policy [32•]. However, compliance with masking may be difficult to measure and enforce. Where penalties are used, the type varies from institution to institution. Some terminate employees that do not receive a vaccine or get an approved exemption $[33,34]$. One academic medical center linked suboptimal vaccination rates in academic departments to loss of good standing with the dean and withholding of budget allocations [30].

Exemptions from vaccination also vary between institutions and include medical contraindications, such as allergy to the vaccine or history of Guillain-Barré following influenza vaccination, religious beliefs, or other personal or philosophical reasons. In the 2017 survey, among the 368 hospitals with a vaccine mandate, $96.2 \%$ reported that they allowed medical exemptions, $78 \%$ allowed religious exemptions, and 7.9\% allowed other reasons for exemptions [32•]. Twelve point eight percent of the hospital survey did not require a specific reason for exemptions. There is no best practice offered as to who should review the requests for exemption and determine whether to grant them. In one study, medical 
and religious exemptions were allowed, and all HCP that requested them were evaluated in the occupational health office [34]. In another study, requests for religious exemptions were handled by human resources and requests for medical exemptions required an accompanying letter from a licensed physician [33].

Mandatory vaccine policies have been shown to reliably increase the rate of HCP vaccination [11, $30,33,34]$. Healthcare facilities with mandatory policies have higher HCP vaccination rates than those without a mandatory policy [25]. Even in settings in which other methods have been used to increase vaccination rates, requiring non-vaccinated employees to wear masks during influenza season may be associated with an increase in vaccine uptake. In one German hospital, a masking policy for non-vaccinated employees led to an improvement in vaccination rate from 33 to $51.7 \%$ in the first 10 days after implementation of the policy [35]. Feeling uncomfortable while wearing a mask was associated with increased odds of getting vaccinated [29]. Other consequences for non-vaccination also influence HCP vaccination rates. A linear model estimated that termination for noncompliance increased vaccination rates by 12.8 percentage points and consequences other than termination by 11.5 percentage points [36]. In the study where vaccination rates were linked to budget allocations in academic departments, that policy increased the vaccination rates among HCP in the school of medicine from 77.5 to $93.7 \%$ [30].

In the 2017-2018 influenza season, vaccine coverage among HCP with an employer vaccination requirement reached $94.8 \%$ [28•]. There has been an increase in the number of healthcare systems in the USA with mandatory vaccine policies [32•], and now all Veteran's Administration Hospitals have mandatory vaccine policies. In a survey by the CDC of the 2017-2018 influenza season, $44.1 \%$ of responding HCP reported having an employer vaccination requirement [28•]. Only one single-center study looked at the effect on patient outcomes. They found a decrease in nosocomial influenza rates with increasing HCP vaccination rates [11]. However, this study looked at multiple methods to increase vaccination rates over time, with the vaccine mandate coming fairly late in the timeline, and the decrease in nosocomial influenza rates started prior to the vaccine mandate. No study looking just at the effect of mandatory vaccine policies has studied patient outcomes following mandatory vaccination.

In conjunction with facility policies, eighteen states have now established vaccination requirements for hospital HCP. ${ }^{37}$ These requirements vary based on the hospital type and the type of requirement. Ten states have vaccination assessment requirements, eleven states require hospitals to offer influenza vaccination, eight states require hospitals to ensure HCP are vaccinated, and three states require HCP who decline or are exempted from vaccination to wear masks [37]. When these policies have been studied in combination, facility mandates alone lead to more of an increase in vaccination rates than state laws alone, but the combination has an additive effect [38].

In general, there has been high acceptance for mandatory vaccine policies among $\mathrm{HCP}$, greater than $70 \%$ in several surveys $[39,40]$. Interestingly in one study, while $72 \%$ of respondents felt that vaccine mandates were coercive, 95\% felt they were important for protecting patients and staff and more than $90 \%$ felt that it was part of the job responsibilities [40]. When HCP were surveyed about mask-wearing policy during the 2009 pandemic influenza season, $70 \%$ considered the policy to be justifiable $[29,41]$, similar to the acceptance for mandatory vaccine policies. However, over $58 \%$ in one survey [29] and $69.1 \%$ in another survey [41] felt that mask wearing was uncomfortable, which can influence the decision around receipt of vaccine. However, mandatory vaccine policies are not universally welcomed and there have been legal challenges to such policies. Institutions that wish to pursue mandatory influenza vaccine policies should consider making it a condition of employment included in contracts [42].

\section{Conclusion}

Influenza vaccination of HCP remains an important initiative to protect $\mathrm{HCP}$ and their patients. Despite decades of recommendations, national rates remain below the Healthy People 2020 goal overall [3]. Facilities that have instituted 
mandatory programs have consistently high vaccination rates, but challenges remain in long-term care and ambulatory settings.

\section{Compliance with Ethical Standards}

\section{Conflict of Interest}

Megan C Gallagher declares that she has no conflict of interest.

Sarah Haessler declares that she has no conflict of interest.

Hilary M Babcock declares that she has no conflict of interest.

\section{Human and Animal Rights and Informed Consent}

This article does not contain any studies with human or animal subjects performed by any of the authors.

\section{References and Recommended Reading}

Papers of particular interest, published recently, have been

highlighted as:

- Of importance

1. Grohskopf LA, Alyanak E, Broder KR, et al. Prevention and control of seasonal influenza with vaccines: recommendations of the advisory committee on immunization practices-United States, 2019-20 influenza season. Morb Mortal Wkly Rep Recomm Rep. 2019;68(RR-3):1

The ACIP recommendations on seasonal influenza vaccination are updated yearly and are an important resource for current vaccination practices.

2. Shefer A, Atkinson W, Friedman D, et al. Immunization of health-care personnel: recommendations of the advisory committee on immunization practices (ACIP). Morb Mortal Wkly Rep Recomm Rep. 2011;60(RR-7):1.

3. Immunization and Infectious Diseases. US Office of Disease Prevention and Health Promotion website. https://www.healthypeople.gov/2020/topicsobjectives/topic/immunization-and-infectiousdiseases/objectives. Accessed September 24, 2019.

4. Rolfes MA, Flannery B, Chung JR, et al. Effects of influenza vaccination in the United States during the 2017-2018 influenza season. Clin Infect Dis. 2019;69:1845. https://doi.org/10.1093/cid/ciz075.

5. Ray GT, Lewis N, Klein NP, Daley MF, Wang SV, Kulldorff $\mathrm{M}$, et al. Intraseason waning of influenza vaccine effectiveness. Clin Infect Dis. 2019;68(10):1623-30.

6. Wilde JA, McMillan JA, Serwint J, et al. Effectiveness of influenza vaccine in health care professionals: a randomized trial. JAMA. 1999;281(10):908-13.

7. Potter J, Stott DJ, Roberts MA, Elder AG, O'Donnell B, Knight PV, et al. Influenza vaccination of health care workers in long-term-care hospitals reduces the mortality of elderly patients. J Infect Dis. 1997;175:1-6.
8. Carmen WF, Elder AG, Wallace LA, et al. Effects of influenza vaccination of health-care workers on mortality of elderly people in long-term care: a randomised controlled trial. Lancet. 2000;355:93-7.

9. Hayward AC, Harling R, Wetten S, et al. Effectiveness of an influenza vaccine programme for care home staff to prevent death, morbidity, and health service use among residents: cluster randomised controlled trial. BMJ. 2016;333:1241. https://doi.org/10.1136/bmj. 39010.581354 .55 .

10. Salgado CD, Giannetta ET, Hayden FG, Farr BM. Preventing nosocomial influenza by improving the vaccine acceptance rate of clinicians. Infect Control Hosp Epidemiol. 2004;25:923-8.

11. Frenzel E, Chemaly RF, Ariza-Heredia E, et al. Association of increased influenza vaccination in health care workers with a reduction in nosocomial influenza infections in cancer patients. Am J Infect Control. 2016;44:1016-21.

12. Amodio E, Restivo V, Firenze A, Mammina C, Tramuto F, Vitale F. Can influenza vaccination coverage among healthcare workers influence the risk of nosocomial influenza-like illness in hospitalized patients? J Hosp Infect. 2014;86:182-7.

13. Bénet $\mathrm{T}$, Régis $\mathrm{C}$, Voirin $\mathrm{N}$, et al. Influenza vaccination of healthcare workers in acute-care hospitals: a casecontrol study of its effect on hospital-acquired influenza among patients. BMC Infect Dis. 2012;12:30 http://www.biomedcentral.com/1471-2334/12/30.

14. Gudgeon P, Wells DA, Baerlocher MO, Detsky AS. Do you come to work with a respiratory tract infection? Occup Environ Med. 2009;66:424. 
15. Jena AB, Baldwin DC, Daugherty SR, Meltzer DO, Arora VM. Presenteeism among resident physicians. JAMA. 2010;304(11):1166-8.

16. Turnberg W, Daniell W, Duchin J. Influenza vaccination and sick leave practices and perceptions reported by health care workers in ambulatory care settings. Am J Infect Control. 2010;38:486-8.

17. Jena AB, Meltzer DO, Press VG, Arora VM. Why physicians work when sick. Arch Intern Med.

2012;172(14):1107-8.

18. Babcock HM, Beekmann SE, Pillai SK, et al. Reported variability in healthcare facility policies regarding healthcare personnel working while experiencing influenza-like illnesses: an emerging infections network survey. Infect Control Hosp Epidemiol. https:// doi.org/10.1017/ice.2019.305.

19. O'Neil CA, Kim L, Prill MM, et al. Respiratory viral surveillance of healthcare personnel and patients at an adult long-term care facility. Infect Control Hosp Epidemiol. 2019;40:1390-12.

20. Blake KD, Blendon RJ, Viswanath K. Employment and compliance with pandemic influenza mitigation recommendations. Emerg Infect Dis. 2010;16(2):212-8.

21. Leung NHL, Xu C, Ip DKM, Cowling BJ. The fraction of influenza virus infections that are asymptomatic: a systematic review and meta-analysis. Epidemiology. 2015;26(6):862-72.

22. Foy MH, Cooney MK, Allan ID, Albrecht JK. Influenza $\mathrm{B}$ in households: virus shedding without symptoms or antibody response. Am J Epidemiol. 1987;126(3):506-15.

23. Patrozou E, Mermel LA. Does influenza transmission occur from asymptomatic infection or prior to symptom onset? Public Health Rep. 2009;124:193-6.

24. Murti M, Otterstatter M, Orth A, Balshaw R, Halani K, Brown PD, et al. Measuring the impact of influenza vaccination on healthcare worker absenteeism in the context of a province-wide mandatory vaccinate-ormask policy. Vaccine. 2019;37:4001-7.

25. Frederick J, Brown AC, Cummings DA, et al. Protecting healthcare personnel in outpatient settings: the influences of mandatory versus nonmandatory influenza vaccination policies on workplace absenteeism during multiple respiratory virus seasons. Infect Control Hosp Epidemiol. 2018;39(4):452-61.

26. Saxen H, Virtanen M. Randomized, placebo-controlled double blind study on the efficacy of influenza immunization on absenteeism of health care workers. Pediatr Infect Dis J. 1999;18(9):779-83.

27. Sartor C, Zandotti C, Romain F, Jacomo V, Simon S, Atlan-Gepner C, et al. Disruption of services in an internal medicine unit due to a nosocomial influenza outbreak. Infect Control Hosp Epidemiol. 2002;23(10):615-9.

28. $\quad$ Black CL, Yue X, Ball SW, et al. Influenza vaccination coverage among health care personnel-United States, 2017-2018 influenza season. Morb Mortal Wkly Rep. 2018;67(38):1050-4
This survey provides data on the current rates of influenza vaccine coverage among HCP.

29. Dorribo V, Lazo-Blanchet C, Hugli O, Zanetti G. Health care workers' influenza vaccination: motivations and mandatory mask policy. Occup Med. 2015;65:739-45.

30. Quan K, Tehrani DM, Dickey L, Spiritus E, Hizon D, Heck K, et al. Voluntary to mandatory: evolution of strategies and attitudes toward influenza vaccination of healthcare personnel. Infect Control Hosp Epidemiol. 2012;33(1):63-70.

31. Drees M, Wroten K, Smedley M, Mase T, Schwartz JS. Carrots and sticks: achieving high healthcare personnel influenza vaccination rates without a mandate. Infect Control Hosp Epidemiol. 2015;36(6):717-24.

32. Green MT, Fowler KE, Ratz D, et al. Changes in influenza vaccination requirements for health care personnel in US hospitals. JAMA Netw Open 2018;1(2):e180143, doi:https://doi.org/10.1001/ jamanetworkopen.2018.0143

This survey provides an overview of vaccine policies and requirements across the US.

33. Babcock HA, Gemeinhart N, Jones M, Dunagan WC, Woeltje KF. Mandatory influenza vaccination of health care workers: translating policy to practice. Clin Infect Dis. 2010;50:459-64.

34. Karanfil LV, Bahner J, Hovatter J, Thomas WL. Championing patient safety through mandatory influenza vaccination for all healthcare personnel and affiliated physicians. Infect Control Hosp Epidemiol. 2011;32(4):375-9.

35. Wicker S. Unvaccinated health care workers must wear masks during flu season-a possibility to improve influenza vaccination rates? Vaccine. 2009;27:2631-2.

36. Zimmerman RK, Lin CJ, Raymund M, Bialor J, Sweeney PM, Nowalk MP. Hospital policies, state Laws, and healthcare worker influenza vaccination rates. Infect Control Hosp Epidemiol. 2013;34(8):854-7.

37. Centers for Disease Control and Prevention (CDC). Public health law program. Menu of state hospital influenza vaccination laws. www.cdc.gov/phlp/docs/ menu-shfluvacclaws.pdf. Published 2017. Accessed Sept 4, 2019.

38. Lindley MC, Mu Y, Hoss A, Pepin D, Kalayil EJ, van Santen K, et al. Association of state laws with influenza vaccination of hospital personnel. Am J Prev Med. 2019;56(6):e177-83.

39. Douville LE, Meyes A, Jackson M, Lantos JD. Health care worker knowledge, attitudes, and beliefs regarding mandatory influenza vaccination. Arch Pediatr Adolesc Med. 2010;164(1):33-7.

40. Feemster KA, Prasad P, Smith MJ, et al. Employee designation and health care worker support of an influenza vaccine mandate at a large pediatric tertiary care hospital. Vaccine. 2011;29:1762-179.

41. Brandt D, Rabenau HF, Wicker S. Attitudes of influenza-vaccinated health care workers toward masks to prevent nosocomial transmission of influenza. Influenza Other Respir Viruses. 2011;5(1):61-6. 